\title{
Deploying electronic document management to improve access to hospital medical records
}

\author{
Philip J. Scott and Paul B. Williams
}

\begin{abstract}
There are major problems with the content, structure and management of paper health records. Electronic document management (EDM) has the potential to reduce the costs and risks associated with paper records management. Portsmouth Hospitals NHS Trust has a programme to introduce an EDM solution for health records. The solution has so far been introduced into the ophthalmology and disablement services departments, both of which held separate sets of patient records independent of those in the main records library. This paper discusses the background to the programme and presents the early results, issues and lessons learned so far. The implementation of EDM highlights and magnifies the many problems of historically overstretched records management. Fundamentally, the unsolved question is whether a historical record can be tidied, sorted and indexed sufficiently to make it as usable as the paper record for clinicians in high-volume services. Finding the balance of affordable levels of file preparation to achieve acceptable clinical utility remains a challenge to be resolved.
\end{abstract}

Philip Scott is Head of ICT Development Programmes for Portsmouth Hospitals NHS Trust. He has worked in NHS information management and ICT development projects for 14 years. $\mathrm{He}$ has an MSc with distinction in healthcare information systems and recently completed a doctoral research programme exploring clinician usage of information. He is a Chartered IT Professional and a registered practitioner with the UK Council for Health Informatics Professions. He is a member of the HL7 UK Board and chaired the programme committee for the HL7 UK 2008 conference.

Paul Williams is an independent health informatics consultant who has worked in information management for the past 25 years. He has led several strategic initiatives, including programmes for the Scottish and UK parliaments. He was a thought leader on risk management and information security during the growth of network computing and the use of the internet, providing support to numerous governmental, financial and commercial organisations. For the past four years, he has worked with the Portsmouth NHS community to address the issues of capturing current health records into electronic format. He has an honours degree in chemistry and a PhD in nuclear and radiation chemistry.

Keywords: medical records, document management, electronic health record, managing change

\section{Introduction}

Hospital medical records are fundamental to safe and effective patient care and for subsequent clinical audit, research and medico-legal purposes. However, there are longstanding major problems with the content, structure and management of paper health records.

The 1965 Tunbridge report ${ }^{1}$ on the standardisation of hospital medical records stated that 'Storage space for records is necessarily limited and we were told frequently that the storage of records was a serious and ever growing problem'. Little discernible progress seems to have been made on this issue in the intervening decades. $^{2}$

During 2007, the National Patient Safety Agency received almost 40,000 incident reports related to patient documentation; 36,223 of these were from acute general hospitals. ${ }^{3}$ This suggests either that the NHS hospital sector is the most prone to this type of problem or is more honest about the issues than other care providers. 
A recent investigation of medical record availability estimated that about 1 million outpatient consultations in the NHS each year are conducted without the clinician having access to the patient's notes. ${ }^{4}$ It is anecdotally well known but seldom quantified that junior doctors, nurses and clerical staff collectively spend hundreds of hours finding patient records. ${ }^{5,6}$

The Royal College of Physicians has recognised the need for improvement in hospital medical record content and structure and has issued guidance that covers both operational and clinical standardisation. ${ }^{7,8}$ However, while records remain paper-based, these standards are difficult if not impossible to enforce.

While general practitioners are highly advanced in their use of information technology and 'paperless' working (usually meaning paper-light), only a minority of NHS hospitals have mature corporate electronic patient record (EPR) systems. ${ }^{9,} 10$ The national programme for IT in the English NHS has not yet narrowed this technological divide between the acute and primary care sectors and in any case its scope excludes the management of historical paper records.

Routine adult medical records have to be kept for eight years after the conclusion of treatment (or death), but a significant proportion of records have to be retained for 25-30 years, for example, records involving genetics, maternity, paediatrics, oncology or research trials. ${ }^{11}$ There is also legitimate clinical demand to retain all records of patients with chronic diseases for similar or longer periods as the patient's treatment period is effectively their whole lifetime. Thus, even the introduction of a hospital EPR for newly created records does not in itself quickly remove the need to manage paper notes.

An alternative to maintaining solely paper-based records or a hybrid EPR/paper economy is to implement electronic document management (EDM) for health records. This strategy, either before EPR deployment or in parallel, has the potential to reduce the costs and risks associated with paper records management. NHS acute hospital trusts typically spend at least $£ 1 \mathrm{~m}$ a year on health records management. ${ }^{12,13}$ Implementing EDM should enable major cash-releasing savings to be realised from reduction in administrative staff, records storage space, stationery and transport. It should also lead to efficiency savings from reduced time wastage, safety improvements by enabling multiple concurrent usage of the record and security enhancement by facilitating auditable record access.

Most of the published implementation experience about using EDM for hospital records is from projects in Norway. ${ }^{14-16}$ Portsmouth Hospitals NHS Trust has been developing its EDM strategy since 2005. A previous report described the business case, procurement approach and lessons learned from a pilot deployment. ${ }^{17}$ The present paper reports what has been learned from implementation in ophthalmology and disablement services.

\section{Background}

The ophthalmology department has about 100 staff. Its principal services are outpatient clinics (over 35,000 appointments a year) and day-case surgery, with a relatively small amount of inpatients and emergency cases. The disablement services department comprises about 25 staff, who manage about 6,000 outpatient attendances a year.

Ophthalmology and disablement services both had independent departmental records libraries before the implementation of the EDM system. Ophthalmology held about 61,000 records and disablement services had about 23,000. 
The EDM solution was deployed against a background of conflicting operational pressures. Trust departments were under pressure to meet the 18-week referral-to-treatment target. The administrative function within ophthalmology was under considerable stress as there had been a reduction in the numbers of substantive clerical and administrative staff in the weeks leading up to the EDM deployment, and several agency staff were filling key posts in the department. This inevitably had a detrimental impact on the efficiency of clerical support functions. This situation was further stressed with the introduction of new administrative processes required for back-office support of the EDM system.

The time pressures and relative priority decisions of clinicians were reflected in the low sign-up for EDM training within the ophthalmology department, with less than 55 per cent going through the formal training process before implementation. Several schemes were attempted to deliver the required training, including drop-in sessions, flexible scheduling of classroom sessions and one-to-one sessions. These measures had little impact on the overall uptake of training in ophthalmology. By contrast, almost all staff in disablement services received classroom training before live use began.

\section{Mixed economy deployment approach}

The number of records scanned in readiness for clinics scheduled for the first few days of live use was limited to ease the impact of deployment. This meant that clinics comprised a mixture of patients with paper-based records and some with EDM records. The aim of this mixed economy approach was to temper the impact of implementation issues in the first few weeks of EDM operation.

Overall, the goal was to ensure that the clinical staff were not swamped with patients with EDM records in the first few days, thus permitting staff to adjust their working patterns more slowly to the use of EDM records. Clinical users would be able to discuss process issues with the EDM team and make simple changes to working practices to accommodate the use of EDM records.

There were concerns that the use of EDM would necessitate a reduction in the number of patients seen in each outpatient clinic. One aim of the mixed economy approach was to minimise the increase in the time taken per patient caused by clinicians being unfamiliar with usage of EDM records.

The gradual introduction of EDM records was also intended to allow the EDM team to give direct support to clinicians during clinics, especially those who had not been available for EDM training before deployment.

\section{Results from ophthalmology}

\section{Preparation and scanning}

A team of EDM clerical staff were placed within the department in order to systematically prepare each ophthalmology record for scanning. Preparation activities included ensuring that material was properly filed within the tab structure of the paper record. All images were removed and were scanned into the EDM record using the trust's in-house scanning capability.

The initial plan was to remove records from ophthalmology at a steady rate over a period of 10-15 weeks. However, operational difficulties as records were being slowly removed from the library led to a more radical approach to the uplift process. The departmental clinic lists were so fluid that it could change by as 
much as 50 per cent over a period of five working days. Uplift plans were amended to speed the removal and scanning of records using a team of EDM programme and scanning personnel.

Given the fluidity of the clinic lists, records could only be uplifted from the departmental library when they were known not to be required for clinics at least ten days in advance. Thus EDM staff could be reasonably certain that records remaining on the shelves would not be required for at least the next ten days (other than for an emergency) and therefore could be removed for scanning.

One functional requirement that was missed in the planning stage but quickly became an operational issue was the use of reports such as visual fields, which in paper format could be easily sorted and compared but once scanned as part of a historical batch were very difficult to use.

A serious process failing by the scanning provider resulted in confusion about which records were available electronically and in loading the required files in time for patient attendances. Confusion about internal procedures also caused delay in scanning temporary paper notes into the EDM system.

A significant error was made in one bulk file uplift that resulted in insufficient pre-scan data preparation, which had serious negative effects on navigability. At present, the overall presentation and usability of scanned historical records is in question and the option of recalling the paper records and only using EDM for new records is under consideration.

\section{Image quality}

Clinicians were initially enthusiastic about the quality of images that were scanned into a separate 'Ophthalmology Historic Images' section of the EDM record. The application enabled users to zoom in on these images and, although these were scanned hard copies, the resolution when enlarged provided clear and usable data. However, the large file size of the high-resolution images meant that they were so slow to load that performance became unacceptable.

Some initial quality problems were experienced with the output of visual field tests that had been printed onto thermographic paper. The contractor responsible for the bulk scanning worked with the EDM team and clinicians to modify the scan preparation and processing parameters to achieve an adequate reproduction of the printed output.

\section{Impact on administrative processes}

The administrative and clerical team in ophthalmology tended to have fixed roles and responsibilities. During the transition phase, an added level of complexity was introduced within the department. New working practices were extensively discussed and documented before deployment and training was provided for the clerical staff. Unfortunately, given the unusually high level of temporary staff, the department struggled to put into practice the agreed process flows. Regular meetings, daily at some points, have been held with clerical and clinical users to resolve operational problems and gather feedback on the use of the system.

A seemingly small but important administrative issue was the production of small quantities of patient labels for use in the clinic. With paper records, a sheet of printed labels was generally available in the folder for the clinician to use on various forms, such as assessment charts or surgical listings. Without a permanent paper file there was nowhere to keep such a sheet of labels, so a whole sheet had to be printed for each patient encounter, clearly very wasteful in both time and stationery costs. It proved surprisingly difficult to put in place a mechanism for printing small batches of patient labels from the trust's patient administration system 
(PAS). This requirement was missed in the planning stage as it was incorrectly assumed that the principal usage of labels was for requesting laboratory tests and that the use of computerised requesting would obviate the need for patient labels.

An additional problem that did not seem to have been accurately anticipated was the volume of scanning cover sheets needed for documents without printed barcodes.

\section{Clinical usability}

Clinicians have remarked that they found it difficult to manage the ergonomics and inter-personal consequences of using a PC screen to read the EDM health record during a patient consultation. Further work is underway to provide guidance on the best placement and usage of screens and keyboards within outpatient clinics. $^{18}$

Performance issues with the EDM application have also negatively impacted on usability and, while some progress has been made, further improvement is required to meet operational time constraints.

The difficulty in getting clinicians involved in the pre-implementation phase of the programme has resulted in several key usability issues not being identified until after operational deployment. Significant reworking of the record structure, EDM navigation model and application presentation is underway at the time of writing to simplify clinician usage.

\section{Patient benefits}

$<$ ful $>$ While clerical and clinical usability problems have legitimately been the prime focus of attention, there have also been examples where patient care has improved directly as result of the system. Within two days of the deployment, three elderly patients presented for clinic appointments only to be told that they had come on the wrong day. Ordinarily, difficulties in finding the health record would have meant that such patients would have been sent home and told to attend on their appointment day. Under EDM, the clerk checked that these patients had records on the system and then arranged for them to be seen by a clinician the same day.

\section{Results from disablement services}

The disablement services uplift and deployment commenced about four weeks after the initial deployment in ophthalmology. Staff at all levels in the department were enthusiastic about the deployment of the EDM system.

\section{Preparation and scanning}

Records held within disablement services are significantly larger than those held by ophthalmology, but were in a good condition and the files were well maintained. The disablement services records were reorganised as part of the preparation for scanning, and the records were given additional structure by adding extra tabs to the files. Clinical and administrative staff were involved in a number of sessions with the EDM team to decide on the new records structure. The goal was to create a file that would be easier to view and access using EDM.

Overall, the uplift and scanning activities for disablement services has proceeded very smoothly. Disruption to core clerical and administrative processes has been minimal.

As with ophthalmology, some difficulty has been experienced as a result of delays in the scanning process. The scanning company, having removed records from the disablement services site, failed to have 
them ready to be loaded into the EDM repository in the agreed time. This in turn meant that records needed to be retrieved urgently from the scanning company as patients presented for treatment.

\section{Impact on administrative processes}

The EDM system has been well received by departmental clerical and administrative staff. The time taken to prepare records for clinics has been greatly reduced: one clerical officer takes between two and three hours to prepare two days of clinics. This activity used to take the full-time efforts of a team of two to three people. Further efficiencies are anticipated as the administrative processes are improved.

As most clinics in disablement services are not consultant-led, there is a need to get non-consultant clinic lists from the PAS into the EDM system. A manual workaround is presently required to enter this information, but automatic information transfer is under development.

\section{Clinical usability}

The EDM system has been well received by the department's clinicians and technicians. There have been no adverse comments about image quality or accessing records through the EDM application. Clinics have not changed in size as a result of the EDM deployment and the use of EDM has had only a marginal impact on the time taken by clinicians and technicians to complete their tasks.

\section{Lessons learned}

The following are the key learning points from the deployments in disablement services and ophthalmology:

- Training: The use of informal training for ophthalmology staff failed to deliver either the coverage (the number of staff trained) or the depth of skill in those that were actually trained. There have been instances where clinical staff have been critical of the capability of the EDM application, but investigation has shown that the issue reflects lack of training rather than functional incapability.

- Interaction with departmental staff: Strong involvement and support at a clinical and administrative level from an early stage is essential to the successful deployment of the system.

- Agreement on process changes: It is essential to secure the informed consent of senior clinical and administrative management to the new processes and key deployment requirements before implementation. Formal sign-off by clinical directors and operational managers will be required for the next stages of deployment.

- Uplift of records: Ensuring that records are scanned into the EDM repository without disrupting clinical activity is a significant challenge if records are moved off-site for scanning. There are various approaches to ensure that the records dispatched are unlikely to be required in the time taken to scan the material. However, there will be instances where a record in the scanning process is required by a clinician. The establishment of a dependable process for the rapid scan and secure transmission of records that are required in an emergency is essential.

- Regular review of supplier processes: The regular review of established supplier processes is essential to ensure that there is no degradation in service quality. 
- Realistic pre-implementation trials: Although trial clinics were conducted before implementation, it has become apparent that they did not realistically reflect actual daily experience in clinic. Particularly in a high-volume outpatient service such as ophthalmology, minor time effects per patient are quickly magnified for a full clinic list.

\section{Conclusions}

The implementation of EDM highlights and magnifies the many problems of historically overstretched records management support and the intrinsic difficulties of extracting meaningful information from poorly structured handwritten notes.

The EDM solution provides clinicians with a complete episodic summary of a patient's history of attendance, taken from the PAS. Through the EDM application, a clinician can quickly see which departments have been or are currently involved with caring for the patient. The value of this functionality was made clear when a patient failed to attend an appointment in disablement services. A quick look at the episode schedule revealed that the patient had suffered a fall and was at that moment under assessment within the emergency department.

The apparently disproportionate effects that seemingly minor process changes can instigate, both positive and negative, illustrate that the acute healthcare environment is a complex adaptive system where the effects of change are often nonlinear. ${ }^{19-21}$ The deployment of EDM with its manifold operational ramifications will inevitably have unintended consequences. ${ }^{22}$ To stand any chance of success, a change programme of this magnitude must therefore take a flexible, adaptive approach rather than a fixed project-oriented methodology. Commercial suppliers sometimes struggle to meet the required level of versatility and agility as their emphasis is understandably on contractual delivery rather than organic change.

Approaches need to be found to release clinicians to be able to contribute more fully during key development phases. An EDM solution is a clinical system that will deliver substantial changes to working practice and as such its deployment must be led by clinical teams.

Current technology cannot emulate the simplicity of reading a paper record. The visual and tactile signposts (colour, texture, handwriting, form type) are unavoidably lost. ${ }^{23}$ This fact must be acknowledged as a present disadvantage of EDM, but balanced against the overall benefits of shared secure access to the record whenever and wherever it is needed.

Clinicians vary sharply in the type of access hardware they believe to be most useful. For example, some strongly promote the use of computers on wheels (COWs) in ward rounds while others feel this is inappropriate. In some areas, such as operating theatres, COWs using wireless networking offer the only practicable way of delivering access to EDM.

While EDM unsurprisingly attracts some vocal resistance, it also acts as a catalyst to articulate the need for improved information sharing and security. EDM is sometimes wrongly perceived as attempting to be an EPR, given that electronic forms for direct data entry are being developed for some functions. However, in reality it can only form part of true EPR functionality that includes services such as electronic requesting, workflow and clinical coding. The trust's strategy is to develop interoperability, integrating clinical information systems to deliver a holistic patient record. 
The goal of this paper is to give an honest assessment of implementing EDM solutions for health records in an acute NHS environment. While the strategic case for a solution that moves paper-based records into an electronic form is compelling, to date the practical difficulties of implementation have delayed their realisation and led the trust to review whether the current approach to scanning historical records is viable. Fundamentally, the unsolved question is whether a historical record can be tidied, sorted and indexed sufficiently to make it as usable as the paper record for clinicians in high-volume services. Previous research, using electronic records, has suggested that while dividing voluminous free-text narratives into segments makes information retrieval easier, too much subdivision makes it harder. ${ }^{24}$ Finding the balance of affordable levels of file preparation to achieve acceptable clinical utility remains a challenge to be resolved.

\section{References}

1. Tunbridge, R. E. (1965) The Standardisation of Hospital Medical Records, HMSO, London.

2. Gainsbury, S. (2008) 'Neglect of records departments puts patient safety at risk', Health Service Journal, 22 May, p. NN.

3. National Patient Safety Agency. (2008). 'Patient safety incident reports in the NHS: National reporting and learning system data summary, Issue 8', available at: http://www.npsa.nhs.uk/patientsafety/patientsafety-incident-data/quarterly-data-reports/ (accessed 12 June 2008).

4. Gainsbury, S. (2008) 'Missing: the notes of more than a million outpatients', Health Service Journal, 22 May, p. N.

5. Revill, J. (2003) 'Patients left as doctors push trolleys', The Observer, 3 August, p. 2.

6. Mitchell, J., Hayhurst, C. and Robinson, S. M. (2004) 'Can a senior house officer's time be used more effectively?', Emergency Medicine Journal, Vol. 21, No. 5, pp. 545-547.

7. Ram, M. B. and Carpenter, I. (2007) 'Why it is essential to go on the record', Health Service Journal, 20 September, p.N.

8. Carpenter, I., Ram, M. B., Croft, G. P. and Williams, J. G. (2007) 'Medical records and record-keeping standards', Clinical Medicine, Vol. 7, No. 4, pp. 328-331.

9. Benson, T. (2002) 'Why general practitioners use computers and hospital doctors do not — Part 2: scalability', British Medical Journal, Vol. 325, No. 7372, pp. 1090-1093.

10. Shaw, N. (2001) Going Paperless: A Guide to Computerisation in Primary Care, Radcliffe, Oxford.

11. Department of Health. (2006) 'Records management: NHS code of practice', available at: http://www.dh.gov.uk/en/Publicationsandstatistics/Publications/PublicationsPolicyAndGuidance/DH_41 31747 (accessed 12 June 2008).

12. McIndoe, R. (2007) 'Time to tear ourselves away from paper', Health Service Journal, 8 March, p. N.

13. Bennington, J. and Bullas, S. (2008) 'Transforming services: health records case study', in Bryant, J. (ed.) Proceedings of Healthcare Computing, BCS Health Informatics Forum, Harrogate.

14. Laerum, H., Karlsen, T. H. and Faxvaag, A. (2004) 'Use of and attitudes to a hospital information system by medical secretaries, nurses and physicians deprived of the paper-based medical record: a case report', BMC Medical Informatics and Decision Making, Vol. 4, Article. 18. Available at http://www.biomedcentral.com/1472-6947/4/18 (accessed 12 June 2008).

15. Laerum, H., Karlsen, T. H. and Faxvaag, A. (2003) 'Effects of scanning and eliminating paper-based medical records on hospital physicians' clinical work practice', Journal of the American Medical Informatics Association, Vol. 10, No. 6, pp. 588-595.

16. Lium, J. T., Laerum, H., Schulz, T. and Faxvaag, A. (2006) 'From the front line, report from a near paperless hospital: Mixed reception among health care professionals', Journal of the American Medical Informatics Association, Vol. 13, No. 6, pp. 668-675.

17. Scott, P. J. and Williams, P. B. (2008) 'Implementing scanned health records in NHS community and hospital services', in Bryant, J. (ed.) Proceedings of Healthcare Computing, BCS Health Informatics Forum, Harrogate.

18. Mann, W. and Slaboch, J. (2004) 'Computers in the exam room - friend or foe?', The Permanente Journal, Vol. 8, No. 4, pp. 49-51.

19. Plsek, P. E. and Greenhalgh, T. (2001) 'Complexity science: The challenge of complexity in health care', British Medical Journal, Vol. 323, No. 7313, pp. 625-628.

20. Shiell, A., Hawe, P. and Gold, L. (2008) 'Complex interventions or complex systems? Implications for health economic evaluation', British Medical Journal, Vol. 336, No. 7656, pp. 1281-1283.

21. Plsek, P. (2001) 'Redesigning healthcare with insights from the science of complex adaptive systems', in Committee on Quality of Health Care in America (eds) Crossing the Quality Chasm, National Academy Press, Washington, DC, pp. 309-22.

22. Harrison, M. I., Koppel, R. and Bar-Lev, S. (2007) 'Unintended consequences of information technologies in health care - an interactive sociotechnical analysis', Journal of the American Medical Informatics Association, Vol. 14, No. 5, pp. 542-549. 
23. Nygren, E. and Henriksson, P. (1992) 'Reading the medical record. I. Analysis of physicians' ways of reading the medical record', Computer Methods and Programs in Biomedicine, Vol. 39, No. 1-2, pp. 112.

24. Tange, H., Schouten, H., Kester, A. and Hasman, A. (1998) 'The granularity of medical narratives and its effect on the speed and completeness of information retrieval', Journal of the American Medical Informatics Association, Vol. 5, No. 6, pp. 571-582. 